DOI: 10.1002/adem.201800492

\title{
Broadband Magnetic Composite Energy Harvester
}

By Mohammed Asadullah Khan, Hanan Mohammed and Jürgen Kosel*

M.A. Khan, H. Mohammed, Prof. J. Kosel*

Computer, Electrical and Mathematical Science and Engineering Division

King Abdullah University of Science and Technology (KAUST)

Thuwal 23955-6900, Saudi Arabia.

E-mail: jurgen.kosel@kaust.edu.sa

\section{Abstract}

Energy from ambient vibrations is a potential source for powering the multitude of sensing and computing systems that comprise the internet of things. In order to exploit the broadband nature of natural low frequency vibrations we present a magnetic composite energy harvester that has a dual resonant response in the sub-100 Hz region. A unique structure composed of a proof mass mounted on an array of high aspect ratio, bioinspired hair like structures called cilia is fabricated using polydimethylsiloxane (PDMS) - NdFeB magnetic microcomposite. This structure has a frequency response comprised of two closely spaced resonant peaks facilitating the desirable broadband behavior at low frequency. Each cilium is shaped like a conical frustum with a top diameter of $200 \mu \mathrm{m}$ and a bottom diameter of $450 \mu \mathrm{m}$ and has $a$ height of $3 \mathrm{~mm}$, while the proof mass is cuboid with dimensions of $12 \mathrm{~mm} \times 12 \mathrm{~mm} \times 8 \mathrm{~mm}$. This composite structure is fabricated on top of a micromachined $1 \mathrm{~cm}^{2}$ planar coil, made up of 40 turns of $7.6 \mu \mathrm{m}$ thick electroplated copper. The effect of material composition of the magnetic composite on the resonant frequencies, bandwidth and energy harvesting performance of the device is studied.

\section{Introduction}

Exponential improvement in performance and power consumption of electronic devices following Moore's law has fueled the rapid growth of the multi-billion dollar market for miniaturized devices ${ }^{[1]}$. This has resulted in miniaturized sensors and actuators enabling 
hitherto unheard-of applications in the fields of personalized healthcare, remote sensing, urban management or wearable devices ${ }^{[2-4]}$. The holy grail of such transduction devices is a completely autonomous, self-contained system. Providing the electrical energy needed for such a system is one of the major roadblocks in attaining this paradigm.

Traditionally, these sensing systems have been powered using electrochemical batteries. However, batteries need to be recharged periodically and they need to be replaced after a certain number of charge-discharge cycles. Depending on the nature of the sensing environment, these operations can be risky, time consuming and economically infeasible. Further, Lithium ion batteries, which are the most commonly used electrochemical cells, can explode catastrophically ${ }^{[5]}$, and also have a pernicious influence on the environment ${ }^{[6]}$. Usually the sensing environment has an abundance of ambient energy in various forms such as electromagnetic radiation (infrared, visible light, cellular signals, etc.), vibrations, temperature gradients, etc. It is particularly attractive to scavenge ambient vibrations for the purpose of powering electronic sensing systems as these are pervasive in most sensing environments in the form of oscillations arising from human/animal locomotion, heat exchange (refrigerators, air conditioning systems), vehicle engines, acoustic signals, etc. ${ }^{[7]}$. Physical phenomena such as electromagnetic induction, magnetoelectricity, piezoelectricity, triboelectricity, etc. are exploited to convert ambient vibrations into electrical energy ${ }^{[8]}$.

Electromagnetic induction harvesters consist of either (a) a moving coil and a static magnetic flux source or (b) a static coil and a moving magnetic flux source. Of these two configurations, the latter one is quite robust as the static electrical contacts are protected from mechanical wear and tear. Thus, electromagnetic harvesters are quite reliable ${ }^{[9]}$ and the preferred energy generation source for large scale electrical installations. Scaling down the electromagnetic harvester has proven to be a difficult endeavor, due to challenges in implementing miniaturized coils and fabricating small permanent magnets. In some designs, the use of a discrete $\mathrm{NdFeB}$ permanent magnet, affixed to the device post the fabrication 
process has been reported ${ }^{[10,11]}$. This is an inelegant solution that is not amenable to mass manufacturing. Some of these harvesters also use wire wound coils, which cannot be miniaturized ${ }^{[12]}$.

Herein, we use a permanent magnetic composite instead of the discrete permanent magnet. The composite material melds together the two desired properties of high flexibility and remanent magnetization, providing a high degree of tune ability in both magnetic properties and geometry ${ }^{[13-18]}$. Also, it is processed at a low temperature $\left(<100^{\circ} \mathrm{C}\right)$ by methods such as spin coating, mold casting and drop casting, which makes it easy to integrate with CMOS processes. A polydimethylsiloxane (PDMS) - iron nanowire composite based energy harvester was reported earlier but it suffers from poor power density $\left(1.98 \mu \mathrm{W} / \mathrm{m}^{3}\right)$ at a high excitation force $(\sim 6.0 \mathrm{~g})^{[19]}$.

An issue with most conventional vibration energy harvesters is that they are single mode devices, which provide maximum output power within a small frequency band centered around the resonant frequency ${ }^{[20]}$. Common vibration sources span a range of frequencies over the entire sub-100 Hz frequency region ${ }^{[7]}$. Therefore, for efficient energy harvesting, it is desirable to design broadband energy harvesters, which can work effectively over a wide range of frequencies.

One approach to tackle the small operating band conundrum is to incorporate magnetic tuning in a piezoelectric cantilever, which results in an energy harvester with configurable resonant frequency ${ }^{[21]}$. In this device, the resonant frequency can be tuned from $22 \mathrm{~Hz}$ to 32 Hz. However, once it is tuned, the frequency response is quite narrow band as expected from such a resonant system. In ${ }^{[22]}$, a single energy harvesting system consisting of multiple series connected cantilevers with different resonant frequencies is presented. This system has a wide operating range of $4.2 \mathrm{kHz}$ to $5 \mathrm{kHz}$, which while too high to harvest ambient mechanical vibrations, might still be used for acoustic energy scavenging. In ${ }^{[23]}$, we see the use of a mechanical stopper, which limits the vibration amplitude and increases the bandwidth of a 
conventional cantilever-proof mass design at the expense of lower peak power. In $^{[24]}$, a nonresonant system comprising of a discrete magnet moving through PCB coils is demonstrated. A tunable dual flexural resonant mode piezoelectric device capable of broadband operation is presented in ${ }^{[25]}$. Also in ${ }^{[26]}$, the analytical model of a broadband magnetoelectric energy harvester using laminated sheets of Terfenol-D and PZT-4 is presented.

In this work, we describe the fabrication and characterization of a broadband energy harvester, which consists of a magnetic composite structure that has two closely spaced resonant peaks, thereby providing a large operating band in the low frequency region that is attractive for vibration energy harvesters. The performance of the energy harvester is evaluated with regards to the particle concentration of the composite.

A schematic of this energy harvester is shown in Fig. 1. It consists of two parts (i) a static planar microfabricated coil and (ii) a flexible magnetic composite structure made using an $\mathrm{NdFeB}$ - PDMS microcomposite. This structure consists of a 10x10 array of high aspect ratio bio-inspired, hair-like structures called cilia loaded with a cuboid proof mass. The low Young's Modulus of PDMS, high remanence of NdFeB, high aspect ratio of the cilia and large proof mass combine to form a structure that is highly elastic and responds readily to low frequency vibrations.

\section{Experimental Section}

\subsection{Fabrication}

The fabrication of the energy harvester involved two steps: (i) Fabrication of planar coil and (ii) NdFeB-PDMS composite preparation and molding of pillar proof-mass system. Fig. 2 depicts the fabrication process in detail.

\subsubsection{Planar Coil Fabrication}

In order to fabricate the planar coil a metal insulator metal three layer process (interconnect layer, dielectric, coil layer) was used. 
A $<100>$ p-type silicon wafer served as the substrate for the device. The substrate was subjected to dry-wet-dry thermal oxidation cycle, which resulted in the growth of a $1.2 \mu \mathrm{m}$ thick silicon dioxide layer. This layer electrically isolated the coil from the substrate.

DC sputtering in argon ambient was used to deposit a $20 \mathrm{~nm}$ thick titanium adhesion layer [27]. Subsequently, a $120 \mathrm{~nm}$ thick layer of platinum was deposited without breaking the vacuum. Photoresist was spun coated on the wafer. This was then baked, exposed using a maskless aligner (Heidelberg Instruments $\mathrm{GmbH} \mu \mathrm{PG}$ 501) and developed to transfer the pattern to the substrate. Dry etching in an argon plasma using the resist as a soft mask formed the interconnect layer.

Plasma enhanced chemical vapor deposition (PECVD) carried out at a temperature of $300^{\circ} \mathrm{C}$ with silane, ammonia and nitrogen as precursors was used to deposit a $150 \mathrm{~nm}$ thick layer of silicon nitride onto the substrate. Throughout this deposition, the plasma frequency was cycled between $13.56 \mathrm{MHz}(14 \mathrm{~s})$ and $300 \mathrm{kHz}(6 \mathrm{~s})$, which lowered the residual stress in the nitride film ${ }^{[27]}$. Photoresist was then coated, exposed and developed to form the required pattern. Using the patterned resist as a soft mask, the nitride layer was subjected to reactive ion etching using the gases $\mathrm{CHF}_{3}$ and $\mathrm{SF}_{6}$. This formed the vias that allow electrical contact between the coil and the interconnect layers.

Subsequently, $20 \mathrm{~nm}$ of titanium was deposited using DC sputtering. Then without breaking the vacuum, $100 \mathrm{~nm}$ of gold was deposited to form the seed layer for electrodeposition of copper. A $9 \mu \mathrm{m}$ thick layer photoresist was spun coated onto the wafer. This was then baked, exposed using the maskless aligner and developed to form the insulating mold, which selectively directs the growth of copper. The wafer was then immersed in a temperature controlled bath of copper sulphate $\left(\mathrm{CuSO}_{4}\right)$ set to $24^{\circ} \mathrm{C}$. Copper was then electroplated onto the seed layer at an average current density of $3.9 \mathrm{~mA} / \mathrm{cm}^{2}$ for 95 minutes, which resulted in the deposition of a $7.8 \mu \mathrm{m}$ thick layer. In order to ensure proper via coverage, pulsed electrodeposition and slow growth rate were used ${ }^{[29,30]}$. 
The photoresist was then removed by thoroughly washing the wafer with acetone and isopropanol. This exposed the underlying seed layer, which was removed by sputter etching in an argon plasma. No mask was used for this etch step. Owing to this, around $200 \mathrm{~nm}$ of the plated copper was also etched along with the seed gold layer. This etch step defined the coil layer with a thickness of $7.6 \mu \mathrm{m}$.

\subsubsection{Composite Fabrication and Molding}

The elastomer PDMS (Sylgard ${ }^{\circ} 184$ ) was prepared by mixing the pre-polymer and the curing agent in the manufacturer recommended 10:1 ratio by weight. A $20 \mu \mathrm{m}$ thick layer of uncured PDMS was deposited on the planar coil by spin coating, and then cured at $90^{\circ} \mathrm{C}$ for 60 minutes. This forms an insulating layer, which provides electrical isolation between the coil and the composite. NdFeB microparticles (Molycorp MQP-16-7FP, mean particle size 5 $\mu \mathrm{m})$ were added to the uncured PDMS in the required proportion. These microparticles were thoroughly incorporated into the PDMS by mechanical stirring.

A $10 x 10$ array of $200 \mu \mathrm{m}$ circles separated by $1 \mathrm{~mm}$ gaps was etched on a $3 \mathrm{~mm}$ thick poly (methyl methacrylate) (PMMA) sheet using a $10.6 \mu \mathrm{m}$ wavelength $\mathrm{CO}_{2}$ laser milling system (Universal® Laser Systems PLS 6.75) to form the mold. Due to the dispersion of the laser beam, the holes in the PMMA mold were not cylinders but conical frustums with a diameter of $200 \mu \mathrm{m}$ at one end and $450 \mu \mathrm{m}$ at the other. The NdFeB - PDMS composite was dispersed onto the coated substrate and the PMMA mold was then mounted on top of it. This assembly was subjected to vacuum desiccation which removed air bubbles from the composite and facilitated proper filling of the holes in the mold. Subsequently it was cured at $90^{\circ} \mathrm{C}$ for $60 \mathrm{~min}$, forming the cilia array depicted in Fig. 2(r). Following a similar procedure, a $1.2 \mathrm{~cm} \times 1.2 \mathrm{~cm} \times 0.8 \mathrm{~cm} \mathrm{NdFeB} \mathrm{-} \mathrm{PDMS} \mathrm{proof} \mathrm{mass} \mathrm{was} \mathrm{also} \mathrm{molded.} \mathrm{The} \mathrm{proof} \mathrm{mass}$ was then bonded to the cilia array using a very thin layer of spin coated PDMS, which concluded the fabrication of the device (Fig. 2(s)). In total, three devices were fabricated with $\mathrm{NdFeB}$ concentrations of $50 \%, 66.67 \%$ and $75 \%$ by weight. 


\subsection{Electrical Characterization Setup}

A Sub Miniature-A (SMA) female connector was soldered directly onto the contact pads of the planar coil to facilitate electrical measurements. In order to characterize the performance of the planar coil, a bare coil without the composite structure on top was placed at the center of a Helmholtz coil setup, which was driven using a combination of a function generator (Agilent 33220A) and a power amplifier (NF Corporation HSA 4012). The resultant a.c. magnetic field was measured using a Gauss Meter (F.W. Bell 6010). The induced voltage in the coil was recorded using a lock-in amplifier (Stanford Research Systems SR850) (Fig. 3(a)).

In order to measure the response of energy harvester to low frequency vibrations, it was mounted on a custom made PMMA stage. This stage was screwed onto an electromagnetic shaker (Tira GmbH 50018), which also had an accelerometer affixed to it. Single tone sinusoidal electrical signals generated by a vibration controller (Tira SVC 01) were amplified by a power amplifier (Tira BAA 60) and this signal was used to drive the electromagnetic shaker. The signals from the accelerometer mounted on the shaker stage were fed back into the vibration controller and used to operate the shaker in constant acceleration or constant displacement mode. The energy harvester output was connected to a low noise preamplifier (Stanford Research Systems SR560). This amplifier had an input impedance (100 M $\Omega$ ) that was 6 orders of magnitude higher than the impedance of the harvester coil, thereby preventing electrical loading and enabling accurate measurements. The amplifier was operated in low pass mode with a cut-off frequency of $1 \mathrm{kHz}$ to ameliorate spurious high frequency noise. The output of the amplifier was fed into a digital storage oscilloscope (Agilent DSO1012A) to visualize and measure the energy harvester performance(Fig. 3(b)).

\section{Results and Discussions}

\subsection{Simulation and Working Principle}


The frequency response of the magnetic composite structure is studied using the FEM software COMSOL. The cilia are modelled as $3 \mathrm{~mm}$ long conical frustums with a bottom diameter of $450 \mu \mathrm{m}$ and a top diameter of $200 \mu \mathrm{m}$. The proof mass is a cuboid of dimensions $1.2 \mathrm{~cm}$ x $1.2 \mathrm{~cm}$ x $0.8 \mathrm{~cm}$. NdFeB-PDMS composites have a density $(\rho)$ greater than $1 \mathrm{gm} / \mathrm{cc}$ and Young's Modulus (E) greater than $1 \mathrm{MPa}$. The precise value of these physical constants depends upon the proportion of $\mathrm{NdFeB}$ in the composite. In order to investigate the effect of these two physical properties of the material on the resonant frequency, Eigen frequencies of this structure are determined while varying the density of the material between $1 \mathrm{gm} / \mathrm{cc}$ and 4 $\mathrm{gm} / \mathrm{cc}$ and the Young's Modulus between $2 \mathrm{MPa}$ and $14 \mathrm{MPa}$. The simulation results show that regardless of the exact values of $\mathrm{E}$ and $\rho$, this structure has two closely spaced resonant modes. The first mode i.e. the T-mode, involves the structure twisting around its center, as depicted in Fig. 4(a) and supplementary S1. The second mode, i.e. the L-mode, involves the translation of the structure parallel to the ground plane (Fig. 4(b), supplementary S2). It is also observed that the resonant frequency is highest for the structure with the highest Young's Modulus and least density and vice versa (Fig. 4 (c)). This dependence is explained as follows: If the stiffness of the structure for a particular resonant mode is $\mathrm{k}$, and the mass is $\mathrm{m}$, then the resonant frequency is given as ${ }^{[31]}$

$$
f^{\prime}=\frac{1}{2 \pi} \sqrt{\frac{k}{m}} .
$$

From the definition of stiffness, it can be shown that the stiffness is proportional to the modulus of elasticity, $\mathrm{E}^{[31,32]}$. Also, it is known that for a structure of fixed volume, the mass is proportional to the density, $\rho$ of the material used to make the structure. Thus, equation (1) can be rewritten as

$$
f^{\prime}=\frac{a^{\prime}}{2 \pi} \sqrt{\frac{E}{\rho}},
$$

where a` depends on the dimensions of the structure and the resonant mode. As expected from equation (2), the Eigen frequencies of the structure, obtained from the simulation, plotted 
against the square root of the ratio of Young's Modulus and density can be fitted by a straight line passing through the origin (Fig. 4(c)).

The composite structure is magnetized in a direction perpendicular to the axis of the cilia i.e. parallel to the planar coil plane. When the structure is displaced along the direction of the magnetization vector, the north pole moves away and the influence of the south pole of the proof mass on the coil increases (Fig. $\mathbf{4 ( d )}$ ). This changes the average magnetic flux density component normal to the coil to a value $\mathrm{B}_{\mathrm{S}}$, which depends upon the displacement and the remanent magnetization of the composite. Similarly, when the structure is displaced in the opposite direction, the coil is subject to a magnetic flux of value $\mathrm{B}_{\mathrm{N}}$. Thus, when this structure is excited by a mechanical vibration of frequency, $\mathrm{f}$, it will also vibrate at the same frequency, producing an a.c. magnetic flux given as

$$
B=\left(\frac{B_{N}-B_{S}}{2}\right) \sin 2 \pi f t=B_{M} \sin 2 \pi f t
$$

According to Lenz's law, the voltage induced in a loop of wire subject to a time varying magnetic flux, $\varphi$, is given as

$$
V=-\frac{d \varphi}{d t}=-A \frac{d B}{d t}
$$

where $\mathrm{B}$ is the magnetic flux density perpendicular to the loop and A is the area enclosed by the loop of wire. For a coil composed of $\mathrm{N}$ turns, the net voltage, VM, produced will be the summation of the voltage induced in each individual turn. Substituting (3) in (4), the magnitude of the voltage induced in the coil, due to the motion of the composite structure, is given as ${ }^{[33]}$

$$
V_{M}=2 \pi f B_{M} \sum_{i=1}^{N} A_{i},
$$

where $A_{i}$ is the area enclosed by the $i^{\text {th }}$ turn of the coil

If the internal resistance of the coil is $\mathrm{R}_{\mathrm{S}}$ and the connected load resistance is $\mathrm{R}_{\mathrm{L}}$, then the root mean square voltage developed across the load is given by 


$$
V_{R M S L}=\frac{V_{M}}{\sqrt{2}}\left(\frac{R_{L}}{R_{S}+R_{L}}\right)=\sqrt{2} \pi f B_{M} \sum_{i=1}^{N} A_{i}\left(\frac{R_{L}}{R_{S}+R_{L}}\right) .
$$

Thereby, the root mean square power delivered to $R_{L}$ by the energy harvester is given by

$$
P_{R M S}=\frac{V_{R M S L}^{2}}{R_{L}}=2\left(\pi f B_{M} \sum_{i=1}^{N} B_{M}\right)^{2} \frac{R_{L}}{\left(R_{S}+R_{L}\right)^{2}} .
$$

\subsection{Magnetic Composite Characterization}

As seen from the SEM image in Fig. 5(a), the NdFeB powder consists of particles with different shapes and sizes of the order of a few microns. When this powder is thoroughly mixed with PDMS, a composite with uniform distribution of magnetic particles is obtained, as shown in Fig. 5(b).

As the magnetic powder is isotropic, it is possible to magnetize the composite in any direction by applying a strong enough magnetic field. The magnetic hysteresis loop for the composite is studied using a SQUID Vibrating Sample Magnetometer (VSM), for different magnetizing fields and the resultant curves are plotted in Fig. 5(c). Below a magnetizing field of $5 \mathrm{kOe}$, the composite does not exhibit any significant amount of remanence. The remanent magnetization increases rapidly with the applied field in the region from $6 \mathrm{kOe}$ to $11 \mathrm{kOe}$, beyond which the slope of this curve reduces (Fig. 5(d)). In this work, the magnetic composite structure is magnetized by applying a $12 \mathrm{kOe}$ field parallel to the coil plane, which results in a remanence to saturation ratio of approximately $60 \%$. Since the performance of magnetic materials degrades with increasing temperature, the remanent magnetization of the composite was studied at temperatures from $-40^{\circ} \mathrm{C}$ to $120^{\circ} \mathrm{C}$. This range encompasses the typical temperatures at which industrial rated electronics are expected to perform reliably ${ }^{[34]}$. As expected, the remanent magnetization of the composite reduces with increasing temperature, and at $120^{\circ} \mathrm{C}$, it falls to $81 \%$ of the value at room temperature $\left(27^{\circ} \mathrm{C}\right)($ Fig. $5(\mathrm{e}))$. 
The effect of material composition on the magnetic characteristics is studied by obtaining the hysteresis curves for the 50\%, 66.67\% and 75\% NdFeB - PDMS composites between -16 kOe and $+16 \mathrm{kOe}$. The resultant curves show that the remanence and saturation magnetization increase with the NdFeB concentration (Fig. 5(f)). This increase is directly correlated with the volume concentration of $\mathrm{NdFeB}$ in the composite. This is expected as the greater the volume of $\mathrm{NdFeB}$ in a unit volume of the composite material, the higher the magnetic flux emanating from it resulting in a greater magnetization. The composition does not have any effect on the coercivity of the material. With a value of approximately $0.58 \mathrm{~T}$, the coercive field is high enough to resist demagnetization, due to stray magnetic fields.

Using an extensiometer (Instron 5966 Universal Testing System), the moduli of elasticity of the composites are found to be $6 \mathrm{MPa}, 8.9 \mathrm{MPa}$ and $12 \mathrm{MPa}$ for the $50 \%, 66.67 \%$ and $75 \%$ NdFeB - PDMS composites, respectively (Supplementary S5). The densities of the 50\%, $66.67 \%$ and $75 \%$ composites are found to be $1.7 \mathrm{gm} / \mathrm{cm}^{3}, 2.2 \mathrm{gm} / \mathrm{cm}^{3}$ and $2.6 \mathrm{gm} / \mathrm{cm}^{3}$, respectively.

\subsection{Electrical Characterization}

Proper operation of the planar coil is verified by subjecting it to ac magnetic field of varying strengths and frequencies using the setup shown in Fig. 3(a). In the first leg of the experiment at a constant frequency, the magnetic field is swept from $0.2 \mathrm{mT}$ to $2.0 \mathrm{mT}$. The experiment is done at 3 frequencies i.e. $35 \mathrm{~Hz}, 65 \mathrm{~Hz}$ and $95 \mathrm{~Hz}$. The observed induced voltages are plotted (Fig. 6(a)) alongside the analytical results obtained using equation (5). It is seen that there is a linear relationship between the applied magnetic field and the induced voltage and the experimental results agree well with the analytical solutions. Subsequently the frequency is varied from $15 \mathrm{~Hz}$ to $105 \mathrm{~Hz}$, while maintaining a constant magnetic field. The experiment is performed at magnetic field of $1 \mathrm{mT}, 1.5 \mathrm{mT}$ and $2 \mathrm{mT}$. As seen from Fig. 6(b), the measured induced voltage is directly proportional to the frequency of the applied magnetic field and agrees well with the analytical results. 
All three fabricated energy harvesters are tested using the setup shown in Fig. 3(b). The shaker is operated in constant acceleration mode, while sweeping the frequency from $20 \mathrm{~Hz}$ to $100 \mathrm{~Hz}$ and recording the resultant open circuit voltage. The experiment is performed for all three energy harvesters with acceleration values of $1 \mathrm{~g}, 2 \mathrm{~g}$ and $3 \mathrm{~g}$. In all curves (Fig. 7), two resonant voltage peaks corresponding to the two resonant modes of operation are observed. At the first resonant frequency, the vibration of the structure has two components: (i) Motion parallel to the coil plane, due to the stimulus applied by the shaker and (ii) twisting motion about its own center, due to the resonant mode (Supplementary Video S3). Further as the acceleration is increased, the voltage increases proportionately. There is a small increase in resonant frequency of both modes with the $\mathrm{NdFeB}$ concentration. As the $\mathrm{NdFeB}$ concentration of the composite increases, both $\rho$ as well as E increase. Thereby, the E/p ratio, which is closely correlated with the fundamental frequency as well as the mechanical response of the structure, experiences a small increase with increase in concentration of $\mathrm{NdFeB}$ particles. This explains the slight shift to higher values of the resonant frequencies with increasing $\mathrm{NdFeB}$ concentration. It is also observed that the peak output voltage increases significantly with the $\mathrm{NdFeB}$ concentration. A higher resonant frequency is indicative of a stiffer system that has lower vibration amplitude ${ }^{[1]}$. From equation (5), it can be seen that the output voltage depends on the change in magnetic field. The $66.67 \% \mathrm{NdFeB}$ - PDMS composite has $75 \%$ higher remanence than the 50\% NdFeB - PDMS composite and the $75 \% \mathrm{NdFeB}$ - PDMS composite has $144 \%$ higher remanence than the $50 \% \mathrm{NdFeB}-$ PDMS composite. The higher remanent magnetization results in larger magnetic field change and increased induced voltage in devices with more concentration of the magnetic filler.

The load characteristics of the energy harvester are measured by connecting a variable load across the energy harvester and varying it between $20 \Omega$ and $150 \Omega$. The r.m.s. voltage is measured for each resistance value and the rms output power is computed using the expression $\mathrm{P}_{\mathrm{rms}}=\mathrm{V}^{2}{ }_{\mathrm{rms}} / \mathrm{R}_{\mathrm{L}}$. The experiment is performed for all three energy harvesters at the 
first resonant frequency and $3 \mathrm{~g}$ acceleration. As seen in Fig. 8(a), the maximum power in all three cases occurs at a load resistance of $60 \Omega$, which matches the internal impedance of the energy harvesters.

The energy harvesters are subject to $3 \mathrm{~g}$ acceleration, connected to a load of $60 \Omega$, and the voltage is measured, while varying the frequency. The r.m.s. ouput power is computed and plotted against the frequency (Fig. 8(b)). The $3 \mathrm{~dB}$ bandwidth of this energy harvester is the range of frequencies for which the power is at least $50 \%$ of the maximum power obtained at resonance. The maximum power is obtained for the $75 \% \mathrm{NdFeB}$ composition harvester and it is seen that the pass band shifts to the right as the NdFeB concentration increases. It is also seen that in absolute terms the bandwidth increases alongside the NdFeB concentration of the composite. The normalized bandwidth, which is defined as the ratio of the bandwidth to the central pass band frequency, does not vary by much with the composition of the material (Table 1), which is expected as the composite with higher NdFeB concentration has larger bandwidth, but also higher operating frequency.

\section{Conclusions}

In this paper, we have studied the fabrication and characterization of a magnetic composite-based electromechanical energy harvester, capable of wideband energy scavenging in the low frequency regime. The $\mathrm{NdFeB} \mathrm{-} \mathrm{PDMS} \mathrm{microcomposite} \mathrm{features} \mathrm{ease} \mathrm{of}$ fabrication and its low modulus of elasticity is crucial for enabling high performance at low frequencies. The cilia array - proof mass structure enables broadband operation without compromising peak output power. It is also feasible to use this structure with piezoelectric, triboelectric or electrostatic transducers imbuing them with broadband capability, which is a desired trait for multiple applications. While the composition of the magnetic composite does not affect the normalized bandwidth of the energy harvester, it does have a profound effect on the electrical output power of the energy harvester. The normalized bandwidth of this energy harvester is 0.3 , which is among the best results for mechanical energy harvesters (Table 2). 
By virtue of the high filler concentration and low coil resistance, when excited by a $3.0 \mathrm{~g}$ vibration at $42 \mathrm{~Hz}$, the $75 \% \mathrm{NdFeB}$ - PDMS energy harvester delivers a power density of $62.9 \mathrm{~mW} / \mathrm{m}^{3}$, which is 4 orders of magnitude greater than the previous best result for a magnetic composite energy harvester. The power density of the energy harvester can be further improved by using resonant inductive coupling or non-planar coils with higher inductance designed using $3 \mathrm{D}$ printing technologies ${ }^{[35,36]}$.

\section{Supporting Information}

Supporting Information is available online from Wiley InterScience or from the author

\section{Acknowledgements}

Research reported in this publication was supported by the King Abdullah University of Science and Technology (KAUST). The authors also thank the KAUST Nanofabrication and imaging and characterization core laboratory staff at KAUST.

\section{Keywords}

Magnetic Composite, Low Frequency Energy Harvester, Broadband Energy Harvester, $\mathrm{NdFeB}, \mathrm{PDMS}$

Received: ((will be filled in by the editorial staff))

Revised: ((will be filled in by the editorial staff)) Published online: ((will be filled in by the editorial staff))

[1] G. E. Moore, Proceedings of the IEEE 1998, 86, 82.

[2] A. Alfadhel, M. A. Khan, S. C. de Freitas, J. Kosel, IEEE Sensors Journal 2016, 16, 8700.

[3] A. Alfadhel, J. Kosel, Advanced Materials 2015, 27, 7888.

[4] B. Li, O. Yassine, J. Kosel, IEEE Sensors Journal 2015, 15, 453. 
[5] P. Biensan, B. Simon, J. Peres, A. De Guibert, M. Broussely, J. Bodet, F. Perton, Journal of Power Sources 1999, 81, 906.

[6] G. Majeau-Bettez, T. R. Hawkins, A. H. Strømman, Environmental science \& technology 2011, 45, 4548 .

[7] S. Roundy, P. K. Wright, J. Rabaey, Computer communications 2003, 26, 1131.

[8] S. P. Beeby, M. J. Tudor, N. White, Measurement science and technology 2006, 17, R175.

[9] D. P. Arnold, IEEE Transactions on Magnetics 2007, 43, 3940.

[10] R. Amirtharajah, A. P. Chandrakasan, IEEE journal of solid-state circuits 1998, 33, 687.

[11] P. Wang, K. Tanaka, S. Sugiyama, X. Dai, X. Zhao, J. Liu, Microsystem technologies 2009, 15, 941.

[12] A. R. M. Foisal, C. Hong, G.-S. Chung, Sensors and Actuators A: Physical 2012, 182, 106.

[13] A. Alfadhel, B. Li, A. Zaher, O. Yassine, J. Kosel, Lab on a Chip 2014, 14, 4362.

[14] M. Y. Alnassar, Y. P. Ivanov, J. Kosel, Advanced electronic materials 2016, 2, 1600081.

[15] M. A. Khan, A. Alfadhel, J. Kosel, M. Bakolka, in 2016 IEEE Sensors Applications Symposium (SAS), IEEE, 2016.

[16] M. A. Khan, I. R. Hristovski, G. Marinaro, J. Kosel, IEEE Transactions on Magnetics $2017,53,1$.

[17] O. Yassine, A. Zaher, E. Q. Li, A. Alfadhel, J. E. Perez, M. Kavaldzhiev, M. F. Contreras, S. T. Thoroddsen, N. M. Khashab, J. Kosel, Scientific reports 2016, 6, 28539. [18] A. Zaher, S. Li, K. Wolf, F. Pirmoradi, O. Yassine, L. Lin, N. M. Khashab, J. Kosel, Biomicrofluidics 2015, 9, 054113.

[19] M. A. Khan, A. Alfadhel, J. Kosel, IEEE Transactions on Magnetics 2016, 52, 1.

[20] C. Williams, R. B. Yates, sensors and actuators A: Physical 1996, 52, 8. 
[21] V. R. Challa, M. Prasad, Y. Shi, F. T. Fisher, Smart Materials and Structures 2008, 17, 015035.

[22] I. Sari, T. Balkan, H. Kulah, Sensors and Actuators A: Physical 2008, 145, 405.

[23] H. Liu, C. J. Tay, C. Quan, T. Kobayashi, C. Lee, Journal of Microelectromechanical Systems 2011, 20, 1131.

[24] B. Yang, C. Lee, Microsystem Technologies 2010, 16, 961.

[25] P. Li, F. Jin, J. Yang, IEEE Transactions on Ultrasonics, Ferroelectrics, and Frequency Control 2013, 60, 2214.

[26] W. Wang, P. Li and F. Jin, Journal of Physics D: Applied Physics, 51, 1.

[27] M. Todeschini, A. Bastos da Silva Fanta, F. Jensen, J. B. Wagner, A. Han, ACS Applied Materials \& Interfaces 2017, 9, 37374.

[28] A. Tarraf, J. Daleiden, S. Irmer, D. Prasai, H. Hillmer, Journal of Micromechanics and Microengineering 2003, 14, 317.

[29] M. Schlesinger, M. Paunovic, Modern electroplating, Vol. 55, John Wiley \& Sons, 2011.

[30] A. M. Hussain, M. M. Hussain, Small 2016, 12, 5141.

[31] R. K. Shukla, A. Srivastava, in Mechanics, New Age International Pvt. Ltd., Daryaganj, India 2006.

[32] X. Li, T. Ono, Y. Wang, M. Esashi, Applied Physics Letters 2003, 83, 3081.

[33] A. Alfadhel, A. A. A. Carreno, I. G. Foulds, J. Kosel, IEEE Transactions on Magnetics 2013, 49, 4144.

[34] D. Das, N. Pendsé, M. Pecht, L. Condra, C. Wilkinson, IEEE Circuits and Devices Magazine 2000, 16, 26.

[35] C. Ladd, J. H. So, J. Muth, M. D. Dickey, Advanced Materials 2013, 25, 5081.

[36] S. B. Fuller, E. J. Wilhelm, J. M. Jacobson, Journal of Microelectromechanical systems $2002,11,54$. 
[37] H. Xue, Y . Hu, Q. M. Wang, IEEE Transactions on Ultrasonics, Ferroelectrics, and Frequency Control 2008, 55, 2104.

[38] D. S. Nguyen, E. Halvorsen, G. U. Jensen, A. Vogl, Journal of Micromechanics and Microengineering 2010, 20, 125-009-1.

[39] M. S. M. Soliman, E. M. Abdel-Rahman, E. F. El-Saadany, R. R. Mansour, Journal of Microelectromechanical Systems 2009, 18, 1288. 


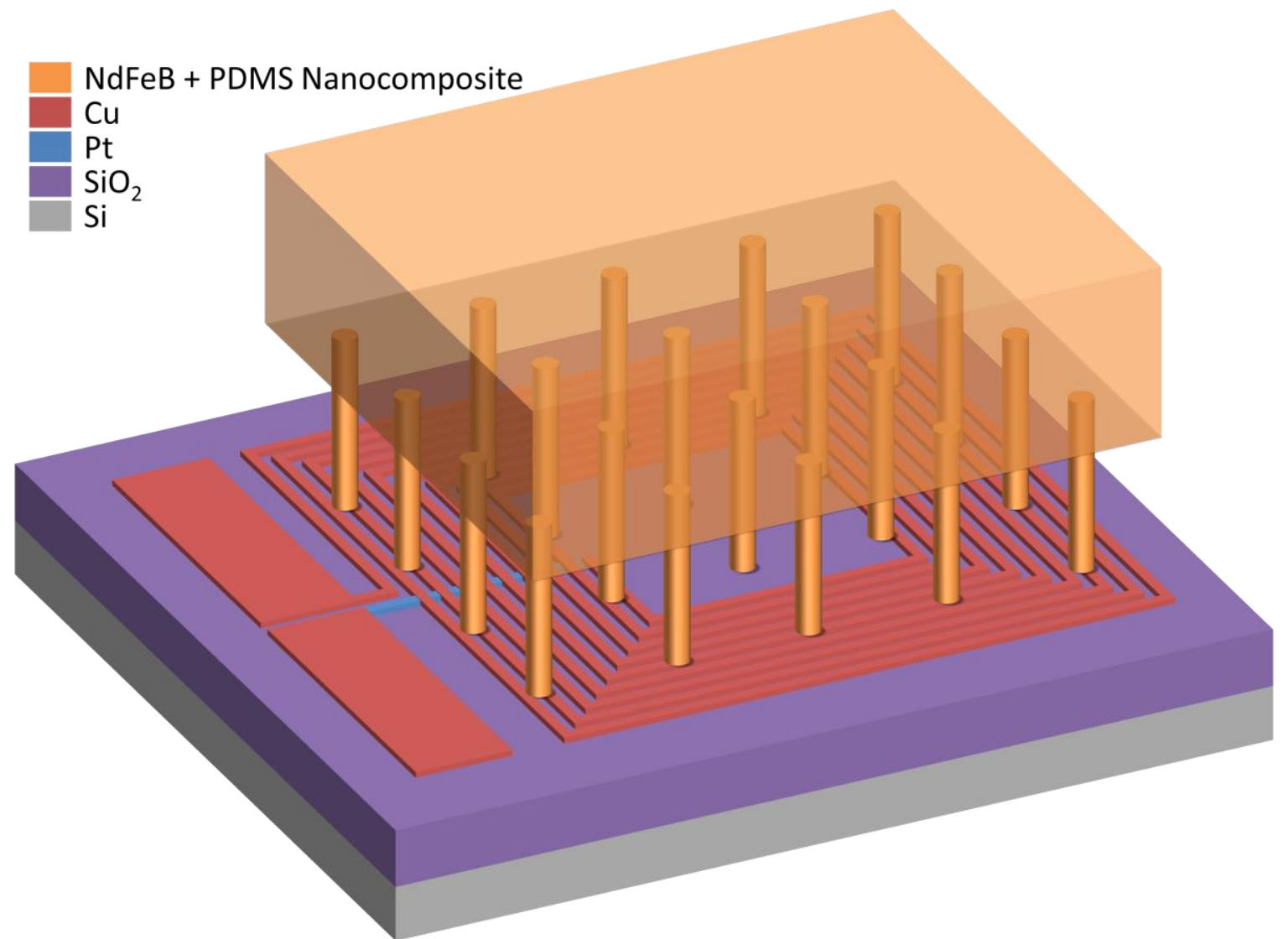

Fig. 1. Schematic of energy harvester 
(a)

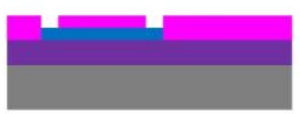

(f)

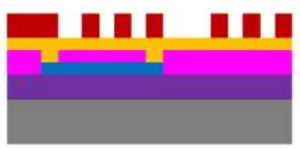

(k)

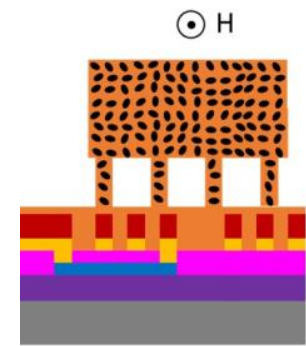

(p)

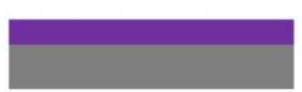

(b)

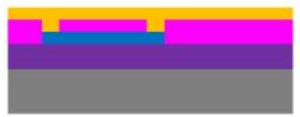

(g)

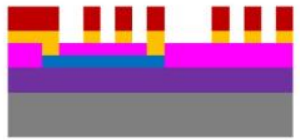

(I)

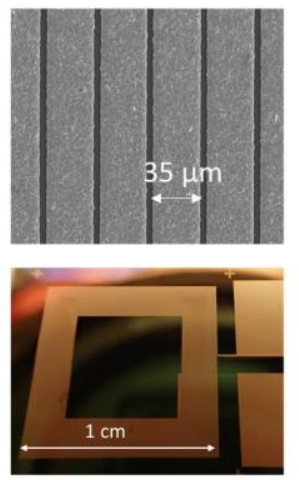

(q)

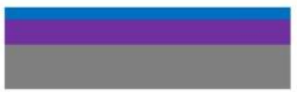

(c)

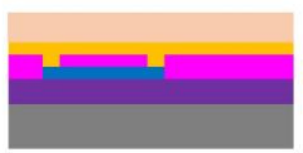

(h)

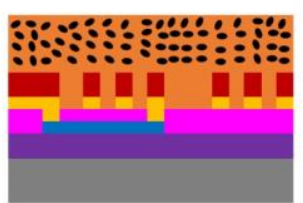

(m)

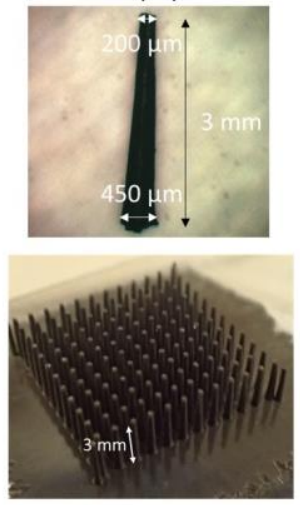

(r)

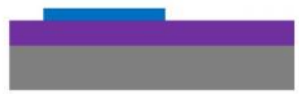

(d)

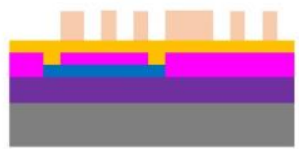

(i)

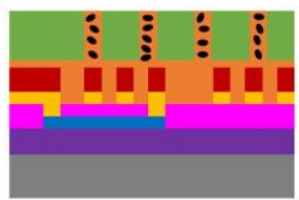

(n)

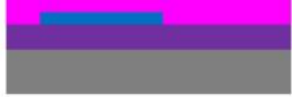

(e)

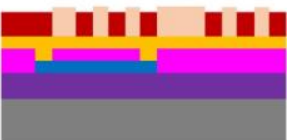

(j)

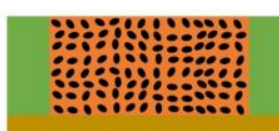

(o)

$\approx \mathrm{Si} \| \mathrm{SiO}_{2} \approx \mathrm{Pt}=\mathrm{SiN}_{\mathrm{x}} \equiv \mathrm{Au}=\mathrm{AZ} 9260=\mathrm{Cu}$ - $\mathrm{PDMS}$ - NdFeB $=\mathrm{PMMA}$

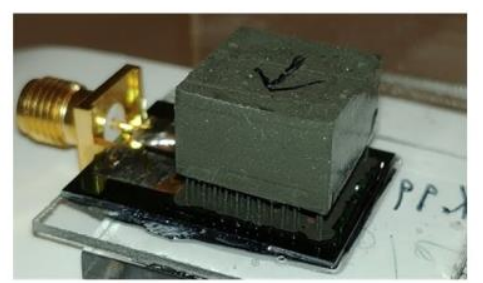

(s)

Fig. 2. Fabrication process flow (not drawn to scale) (a) $\mathrm{Si}$ substrate, (b) $\mathrm{SiO}_{2}$ thermal growth, (c) Pt deposition, (d) Pt dry etch, (e) $\mathrm{Si}_{3} \mathrm{~N}_{4} \mathrm{PECVD}$, (f) $\mathrm{Si}_{3} \mathrm{~N}_{4}$ reactive ion etching, ( $g$ ) Au seed layer deposition, (h) AZ 9260 spin coating, (i) Litho to create mold for electroplating, (j) Cu electroplating, (k) AZ 9260 removal, (l) Au dry etch, (m) NdFeB - PDMS composite drop casting, (n) Composite cilia molding, (o) Composite proof mass molding, (p) Final Assembly and Magnetization, $(q)$ Planar coil array, SEM image of individual turns ( $r$ ) Cilia array and single cilia, $(s)$ Fully assembled energy harvester 


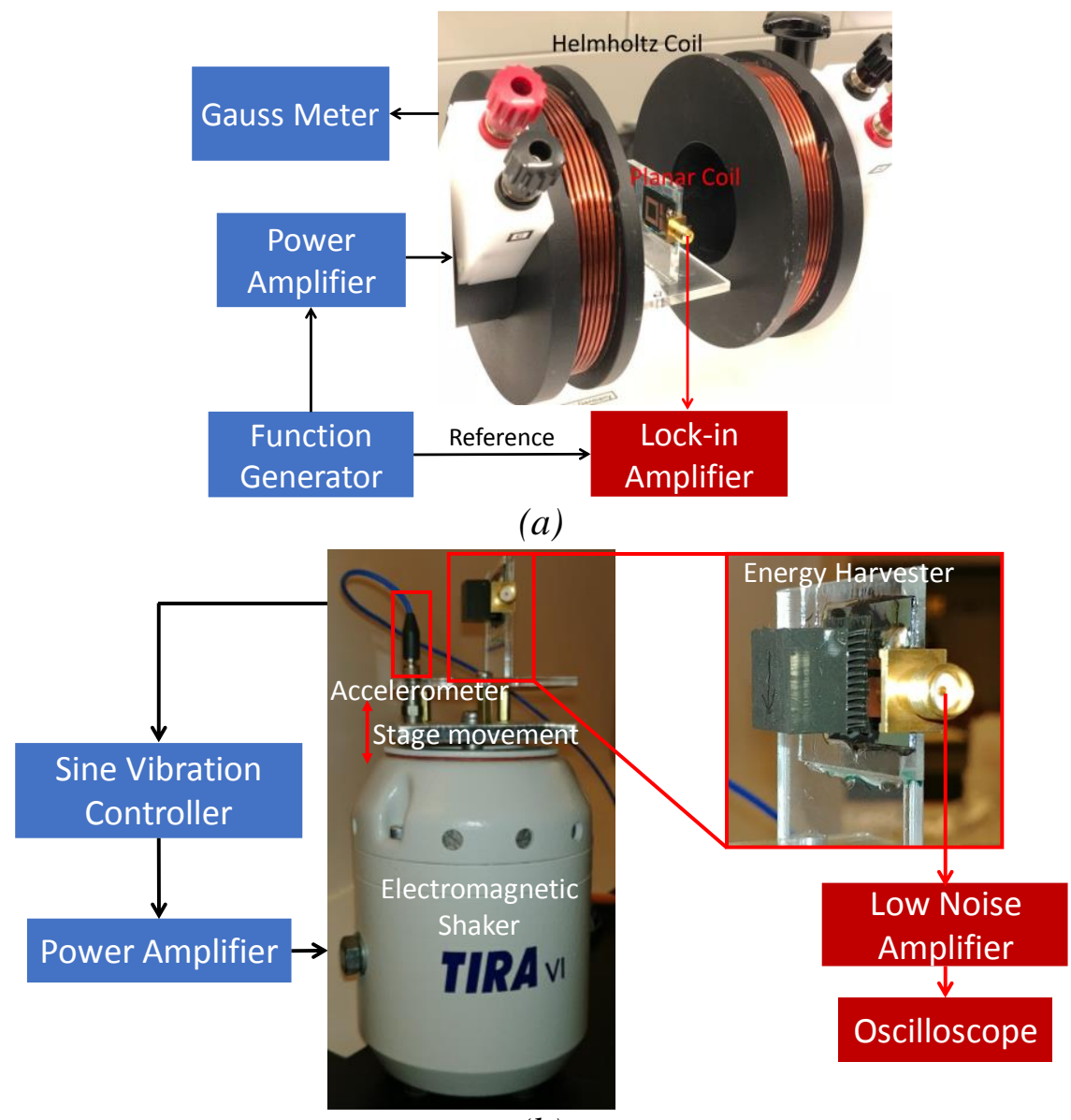

(b)

Fig. 3. Test setup for characterizing (a) the planar coil and $(b)$ the energy harvester 


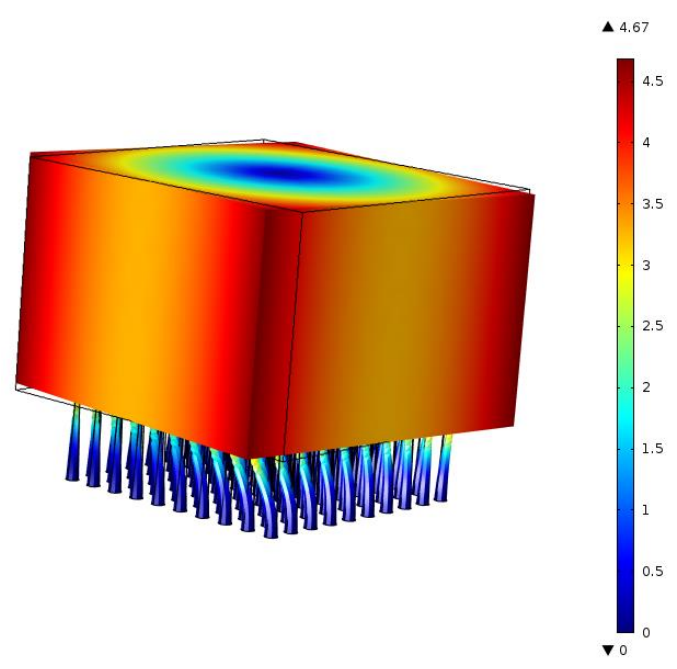

(a)

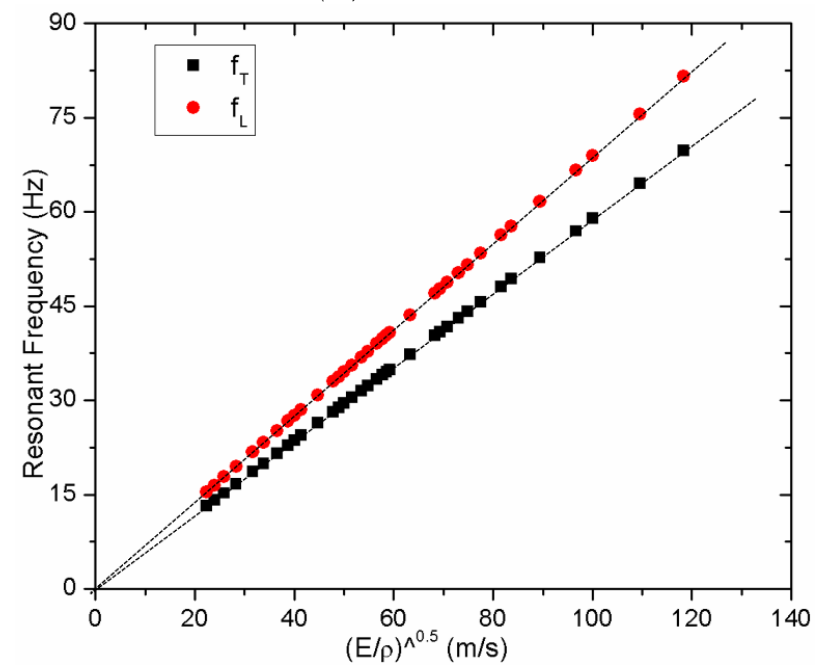

(c)

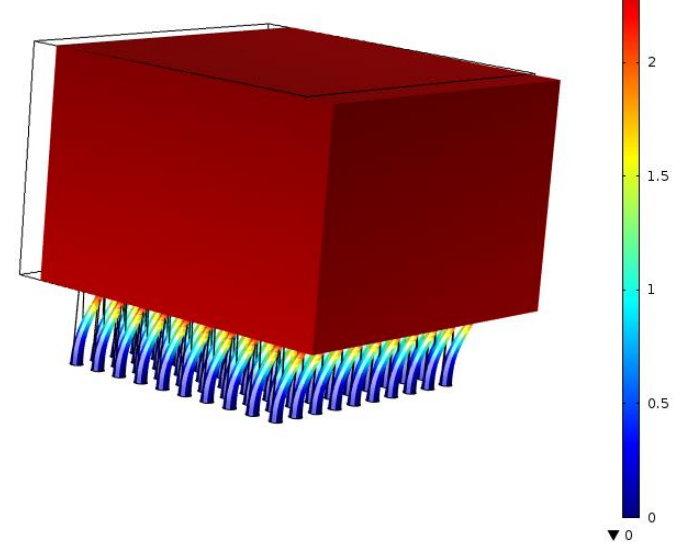

(b)

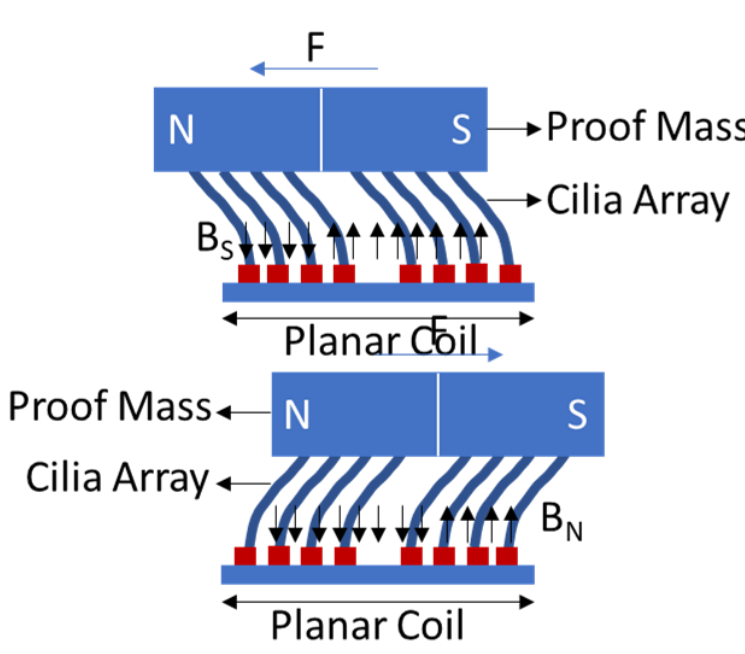

(d)

Fig. 4. Vibration of the resonant harvester structure in (a) T-mode and (b) L-mode (the scale denotes relative displacement in arbitrary units). (c) Dependence of the resonant frequency on the ratio of Young's modulus and density. (d) Harvester operation illustrating the direction of the force applied and the magnetic field component perpendicular to the planar coils 


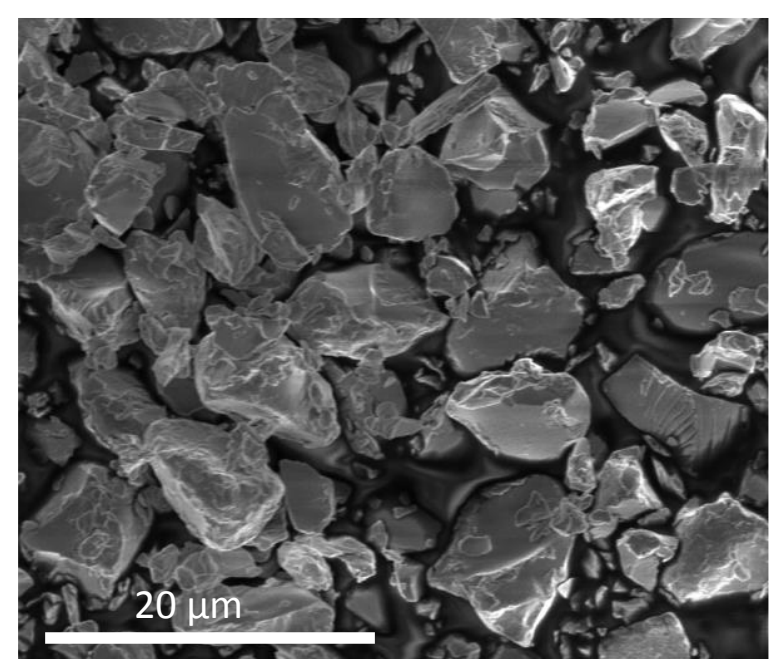

(a)

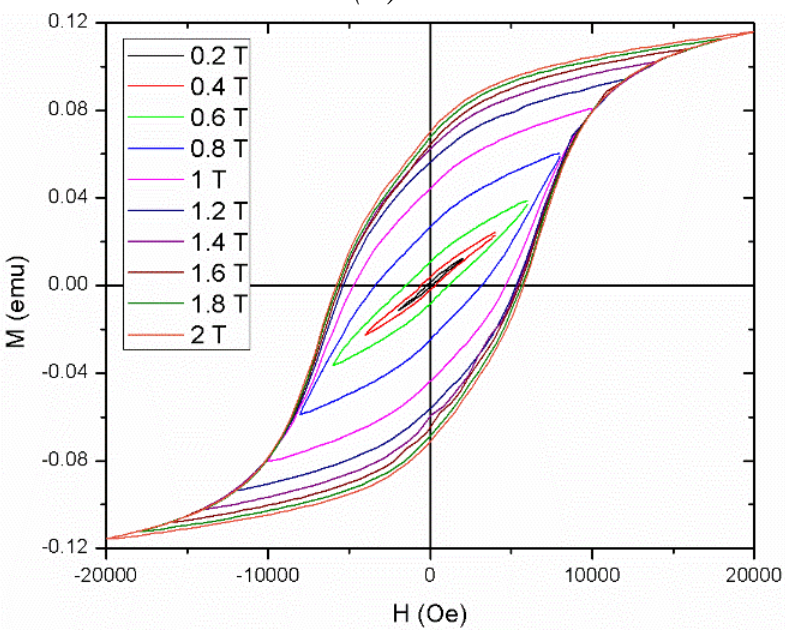

(c)

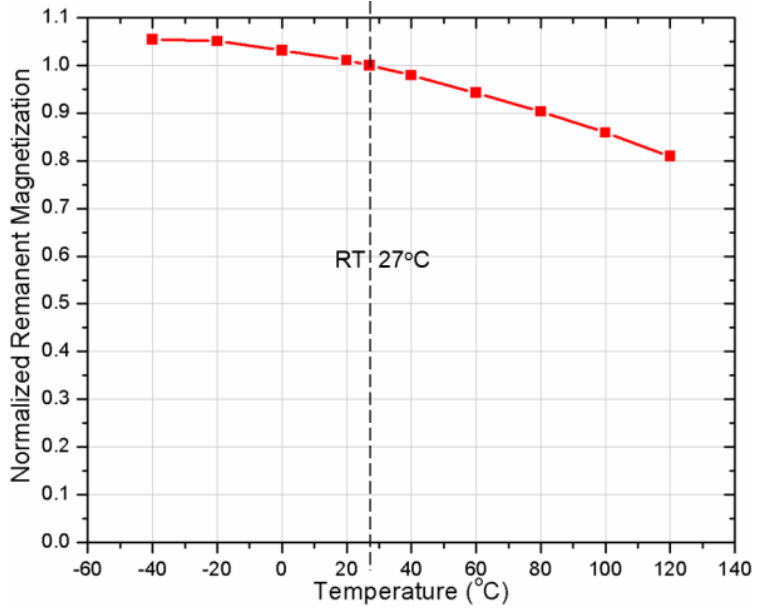

(e)

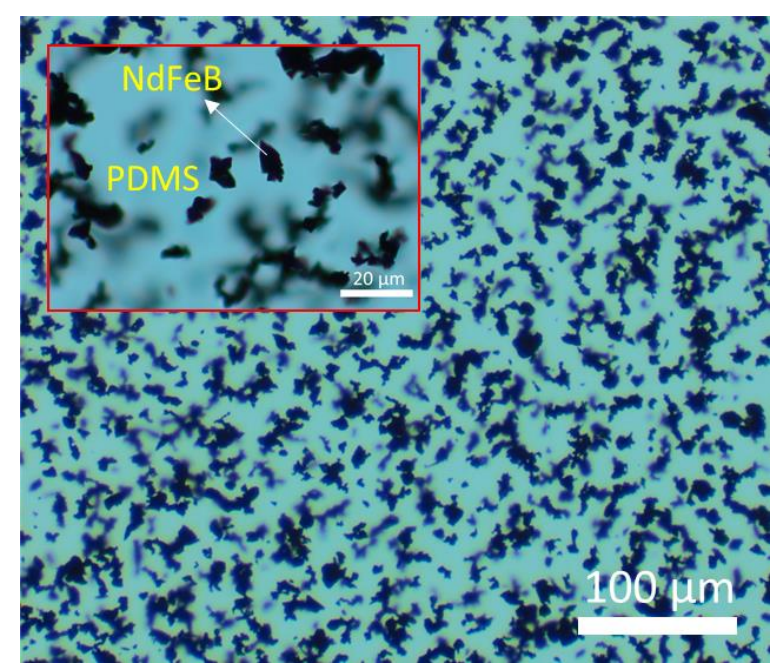

(b)

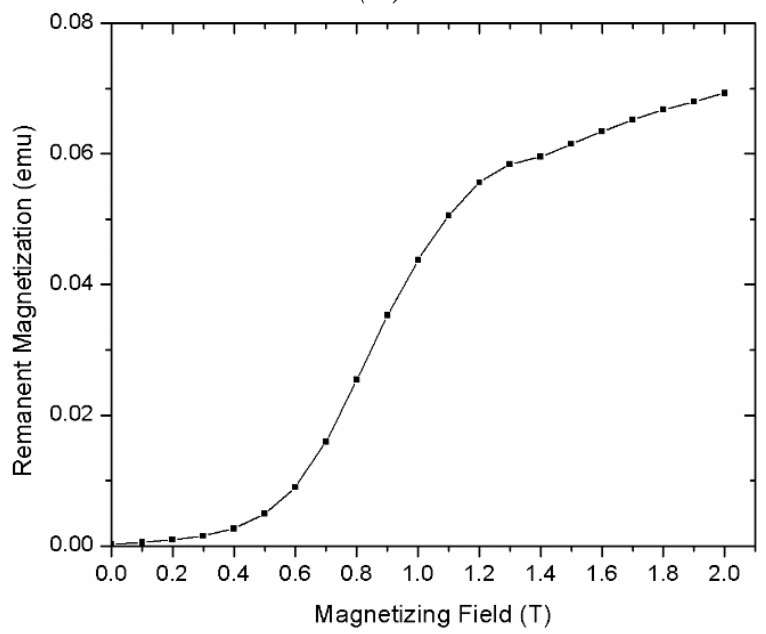

(d)

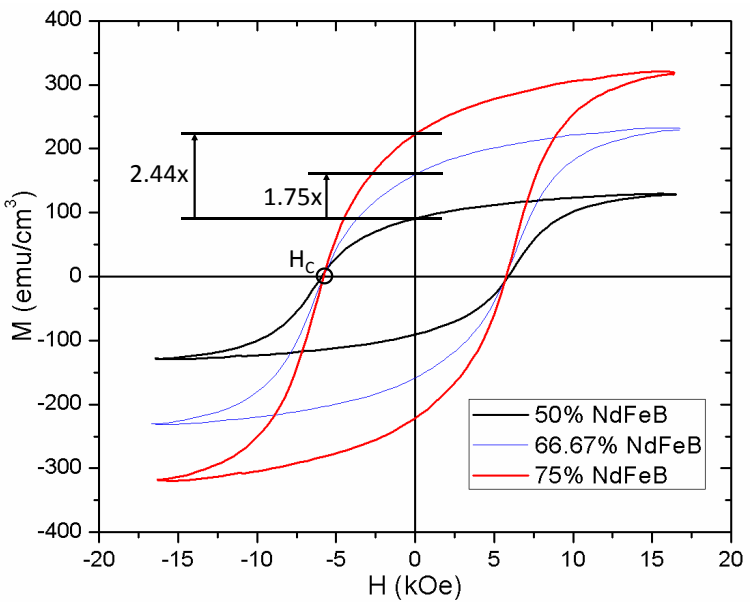

$(f)$

Fig. 5. (a) SEM image of NdFeB powder, (b) Microscopic image of NdFeB - PDMS composite, higher magnification reveals individual NdFeB particles [inset] (c), (d) Variation of magnetization of composite with applied magnetic field, (e) Variation of Remanent Magnetization with temperature. The plot is normalized to the value of $M_{R}$ at room temperature, $(f)$ Hysteresis curves illustrating the effect of filler concentration on remanence and coercivity. 


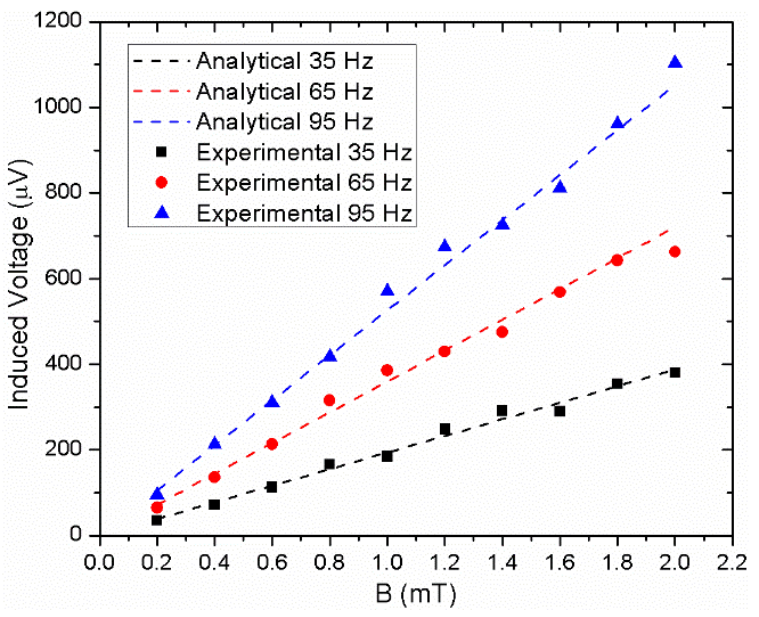

(a)

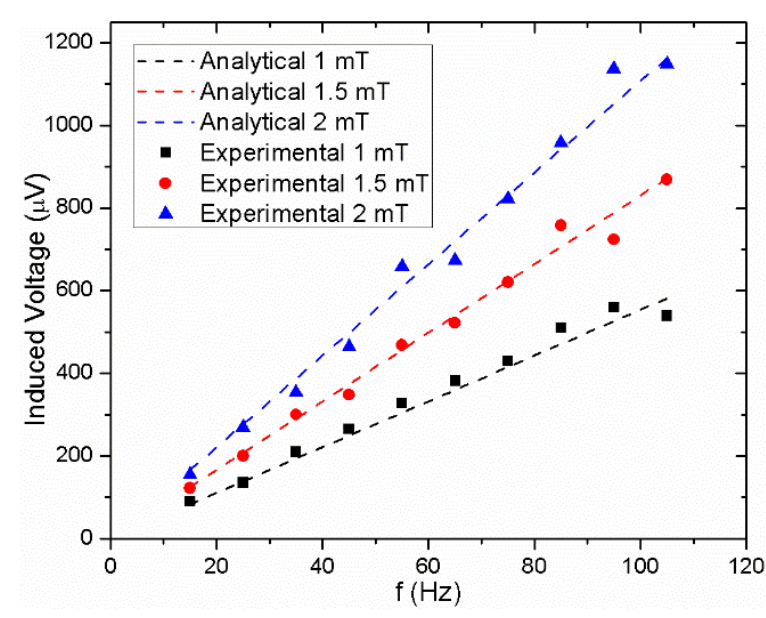

(b)

Fig. 6. Coil induced voltage as a function of (a) Applied Magnetic Field and (b) Frequency. 


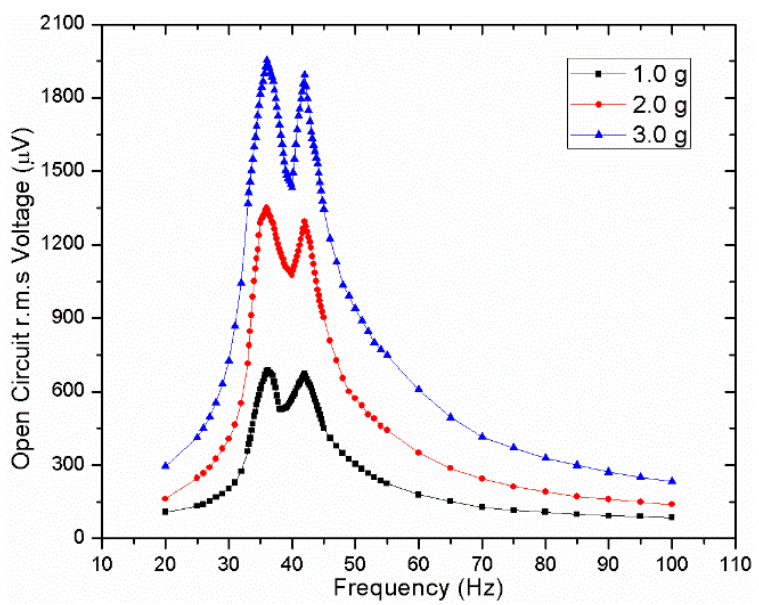

(a)

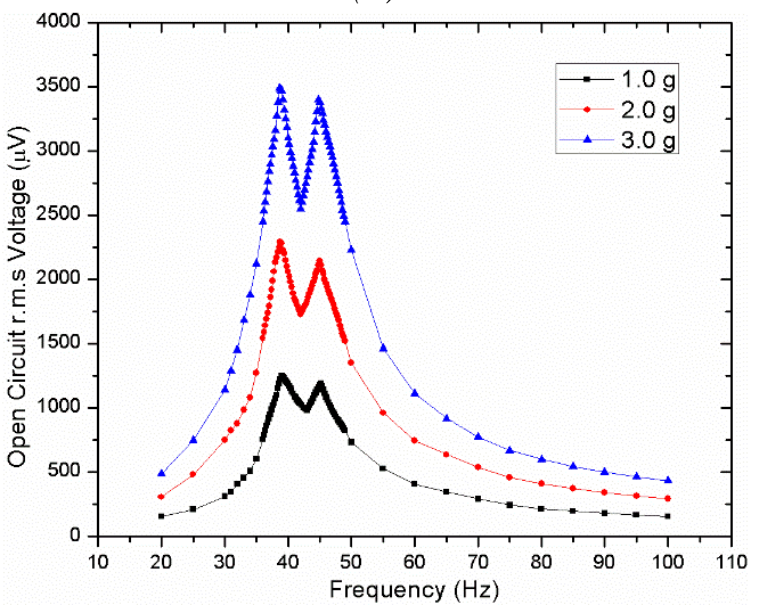

(b)

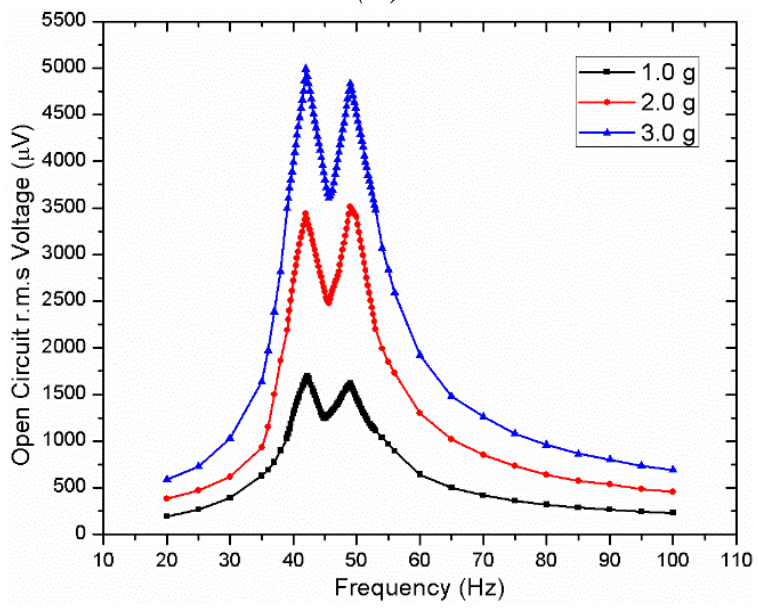

(c)

Fig. 7. Open circuit r.m.s. voltage plotted as a function of frequency at different input acceleration for (a) $50 \% \mathrm{NdFeB}-\mathrm{PDMS}$, (b) $66.67 \% \mathrm{NdFeB}-\mathrm{PDMS}$ and (c) $75 \% \mathrm{NdFeB}-$ PDMS energy harvesters 


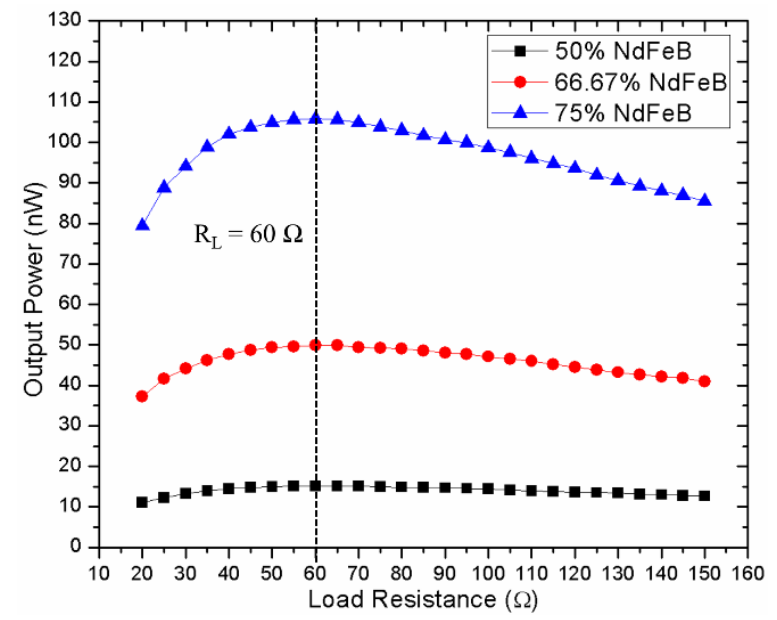

(a)

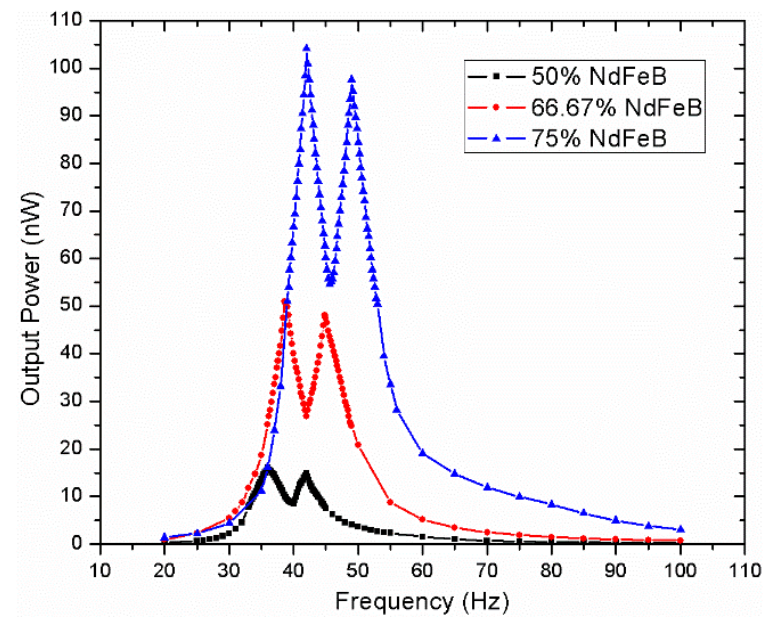

(b)

Fig. 8. (a) Load characteristics of the energy harvesters studied at first resonant frequency and $3 g$ acceleration, (b) Output power of energy harvesters with matched load and $3 g$ acceleration stimulus.

Table 1. Bandwidth and resonant frequency measurements, exp.-experimental, sim.simulation

\begin{tabular}{|c|c|c|c|c|c|c|c|}
\hline \multirow{2}{*}{$\begin{array}{l}\text { NdFeB } \\
\text { Content }\end{array}$} & \multicolumn{2}{|c|}{$\mathrm{f}_{\mathrm{T}}(\mathrm{Hz})$} & \multicolumn{2}{|c|}{$\mathrm{f}_{\mathrm{L}}(\mathrm{Hz})$} & \multirow{2}{*}{$\begin{array}{c}(\mathrm{E} / \rho)^{\wedge} 0.5 \\
(\mathrm{~m} / \mathrm{s})\end{array}$} & \multirow{2}{*}{$\begin{array}{c}\text { Bandwidth } \\
(\mathrm{Hz})\end{array}$} & \multirow{2}{*}{$\begin{array}{l}\text { Normalized } \\
\text { Bandwidth }\end{array}$} \\
\hline & Exp. & Sim. & Exp. & Sim. & & & \\
\hline $50 \%$ & 36 & 35 & 42 & 40.9 & 59.4 & 11.7 & 0.3 \\
\hline $66.67 \%$ & 38.6 & 37.6 & 44.8 & 44 & 63.8 & 12.8 & 0.3 \\
\hline $75 \%$ & 42 & 40.1 & 49.2 & 46.9 & 68 & 13.7 & 0.3 \\
\hline
\end{tabular}

Table 2. Comparison of normalized bandwidths of different broadband energy harvesters

\begin{tabular}{cccc}
$\begin{array}{c}\text { Transduction } \\
\text { Method }\end{array}$ & Mechanical Structure & $\begin{array}{c}\text { Operational } \\
\text { Band }(\mathrm{Hz})\end{array}$ & $\begin{array}{c}\text { Normalized } \\
\text { Bandwidth }\end{array}$ \\
\hline Electromagnetic & Cantilever Array $^{[22]}$ & $4200-5000$ & 0.17 \\
Piezoelectric & Cantilever Array $^{[37]}$ & $87-115$ & 0.28 \\
Electrostatic & Non-linear spring $^{[38]}$ & $520-590$ & 0.13 \\
Electromagnetic & Amplitude Limiter $^{[39]}$ & $94-99$ & 0.05 \\
Electromagnetic & Dual Resonant Structure & $39.1-52.8$ & 0.3 \\
& (Current Work) & & \\
\hline
\end{tabular}

